\title{
JMJD3 promotes SAHF formation in senescent WI38 cells by triggering an interplay between demethylation and phosphorylation of RB protein
}

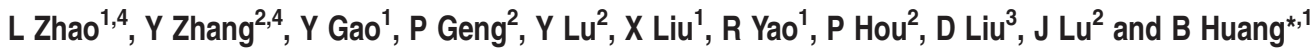

Primary human fibroblasts undergoing oncogene-induced or replicative senescence are known to form senescence-associated heterochromatin foci (SAHF), which can stabilize the state of senescence. The retinoblastoma (RB) protein has an important role in SAHF; cells that lack active RB pathway fail to form SAHF. It has been known that the posttranslational modifications of RB, for example, phosphorylation, regulate its function. To date, whether methylation of RB impacts on the SAHF formation is unknown. Here we report that JMJD3, a histone demethylase catalyzing the tri-methylation of H3K27 (H3K27me3), can demethylate the nonhistone protein RB at the lysine810 residue (K810), which is a target of the methyltransferase Set7/9. We detected a significant upregulation of JMJD3 during cellular senescence and SAHF formation in WI38 cells induced by H-RasV ${ }^{12}$, and we found that ectopic expression of JMJD3 promoted cellular senescence and SAHF formation in WI38 cells. Furthermore, during the process of SAHF assembly, JMJD3 was transported to the cytoplasm and interacted with RB through its demethylase domain JmjC. Significantly, our data demonstrated that the JMJD3-mediated demethylation of RB at K810 impeded the interaction of RB with the protein kinase CDK4 and resulted in reduced level of phosphorylation of RB at Serine807/811 (S807/811), implicating an important role of the interplay between the demethylation and phosphorylation of RB in SAHF assembly. This study highlights the role of JMJD3 as a novel inducer of SAHF formation through demethylating RB and provides new insights into the mechanisms of cellular senescence and SAHF assembly.

Cell Death and Differentiation (2015) 22, 1630-1640; doi:10.1038/cdd.2015.6; published online 20 February 2015

Cellular senescence is an irreversible process of cell cycle arrest. The senescent cells remain metabolically active but are unable to express genes required for cell proliferation. ${ }^{1,2}$ The known causes of cellular senescence include telomere shortening, oxidative stress, DNA damage and hyperoncogenic signaling. ${ }^{3} \mathrm{H}-\mathrm{RasV}^{12}$ has been used as a model to induce senescence in normal cells. ${ }^{4-6}$ Senescent cells are typically characterized by a large flat morphology and the expression of a senescence-associated $\beta$-galactosidase activity (SA- $\beta$-gal), and nuclei of senescence cells may remodel to form the heterochromatin structures termed the senescenceassociated heterochromatin foci $(\mathrm{SAHF}){ }^{7}$ SAHF are condensed regions of DNA that correlate with transcriptionally inactive sites. ${ }^{8}$ These heterochromatin foci are hallmarked by $\mathrm{H} 3 \mathrm{~K} 9 \mathrm{me} 3$ and the incorporation of heterochromatin protein HP1, macroH2A, PML (promyelocy leukemia protein) and HMGA1 (high mobility group AT-hook 1).$^{7,9-11}$ Recently, it has been shown that repressive markers, such as H3K9me3, H3K9me2 and H3K27me3, are rearranged into the nonoverlapping structural layers in SAHF. ${ }^{12-14}$ Changes of heterochromatin organization generate a repressive chromatin environment that prevents transcription of genes that promote growth, thereby stabilize the state of senescence..$^{7,15}$

The retinoblastoma (RB) tumor suppressor is an important senescence regulator, which is typically activated during senescence and enforces the expression of other senescence-inducing proteins. ${ }^{16-18}$ RB protein has a specific E2F-binding domain to suppress E2F activity. ${ }^{19,20}$ Formation of SAHF coincides with the RB tumor suppressor to E2Fresponsive promoters, accompanied with the stable repression of E2F target genes, such as CyclinE, PCNA, MCM4 and CyclinA ${ }^{21,22}$ We found in our earlier study that the activation of RB pathway was necessary for SAHF formation in cancer cells, ${ }^{23}$ and cell lines in which the RB pathway could not be activated failed to form SAHF. Recent studies show that RB can also repress transcription through targeting chromatin remodeling enzymes, for example, histone deacetylases and methyltransferases. ${ }^{24}$ In addition, posttranslational modifications of RB also regulate its activity. Multiple sites of phosphorylation of RB have been identified, and the increased level of $\mathrm{RB}$ phosphorylation at $\mathrm{S} 807 / 811$ results in dissociation of E2F from $R B$ and activates the transcription of genes required for DNA synthesis and cell cycle progression. ${ }^{25}$

${ }^{1}$ The Key Laboratory of Molecular Epigenetics of Ministry of Education, Northeast Normal University, Changchun, China; ${ }^{2}$ The Institute of Genetics and Cytology, Northeast Normal University, Changchun, China and ${ }^{3}$ The Liggins Institute, The University of Auckland, Auckland, New Zealand

${ }^{*}$ Corresponding author: B Huang, The Key Laboratory of Molecular Epigenetics of Ministry of Education, Northeast Normal University, 5268 Renmin Street, Changchun 130024, China. Tel: +86 431 85099798; Fax: +86 431 85099768; E-mail: huangbq705@nenu.edu.cn

${ }^{4}$ These authors contributed equally to this work.

Abbreviations: JMJD3, Jumonji domain containing 3; RB, retinoblastoma; SAHF, senescence-associated heterochromatin foci; Set7/9, SET domain containing protein 7; HMGA1, high mobility group AT-hook 1

Received 31.7.14; revised 08.1.15; accepted 12.1.15; Edited by RA Knight; published online 20.2.15 
Apart from the phosphorylation, methylation of $R B$ has emerged as an important modification in recent years; for example, three lysine residues (K810, K873 and K860) within the C-terminal domain of $\mathrm{RB}$ have been reported to be methylated. ${ }^{25-27}$ Particularly, the methylation of $\mathrm{K} 810$ residue has been found to bring about variable effects on the function of RB. For instance, methylation of $\mathrm{K} 810$ by SET domain containing protein 7 (Set7/9) retains the hypophosphorylated status of RB and suppresses the cellular growth in U2OS cells. ${ }^{28}$ Contrarily, the SMYD2-dependent methylation of RB at K810 promotes cell cycle progression in SW780 and RT4 cells. $^{29}$ The mechanistic details underlying these discrepancies remain unclear. Moreover, whether the methylation of RB impacts the SAHF formation has not been studied prior to this report. In this study, we focused on the effect of RB K810 methylation/demethylation on its function in SAHF formation in WI38 cells.

Jumonji domain containing 3 (JMJD3) is a histone demethylase that demethylates histone $\mathrm{H} 3 \mathrm{~K} 27,{ }^{30}$ and it enhances gene expression by demethylating the repressive H3K27me3 at promoters and gene bodies. As reported, JMJD3 acts as a tumor suppressor through demethylating the H3K27me3 at INK4a/ARF locus, thus increasing the expression of p16 in human and mouse fibroblasts. ${ }^{31,32}$ JMJD3 has also been linked to the regulation of other biological processes, such as differentiation of embryonic stem cells and inflammatory responses in macrophages. JMJD3 is induced during differentiation of glioblastoma stem cells. ${ }^{33}$ JMJD3 can also enhance the nuclear localization of p53 and thus regulate its function. ${ }^{34}$ Moreover, JMJD3 interacts with chromatin modifiers independent of its demethylase activity, to stimulate transcription. ${ }^{30,35}$ However, whether JMJD3 can demethylate non-histone proteins has not been reported prior to this study.

Accumulating evidence has revealed a critical impact of crosstalk between different posttranslational modifications of proteins in various cellular processes. In this study, we present evidence that JMJD3 promotes SAHF formation by demethylating $\mathrm{RB}$ at $\mathrm{K} 810$, which reduce the level of phosphorylation of $\mathrm{RB}$ protein at $\mathrm{S} 807 / 811$, and this interplay promotes the formation of SAHF in WI38 cells. Our findings unravel a novel function of JMJD3 as an inducer of SAHF formation, through demethylating the non-histone protein $\mathrm{RB}$.

\section{Results}

JMJD3 was required for SAHF formation in senescent WI38 cells. To facilitate the study on factors and epigenetic modifiers involved in cellular senescence and SAHF assembly, we first established a cellular model by applying the activated oncogene $\mathrm{H}$-Ras $\left(\mathrm{H}-\mathrm{RasV}^{12}\right)$ to induce senescence and SAHF in human diploid fibroblasts WI38. ${ }^{7}$ Meanwhile, we detected a significant upregulation of JMJD3 mRNA and protein level in WI38 cells following $\mathrm{H}$-RasV ${ }^{12}$ infection for up to 7 days (Figures $1 \mathrm{a}$ and $\mathrm{b}$ ). To determine the functional role of JMJD3 in senescence and SAHF formation, we used the lentivirus-mediated system to silence the JMJD3 expression (Figure 1c). This loss-of-function experiment demonstrated that knockdown of JMJD3 prevented the senescence morphology (Figure 1d) and SAHF formation (Figure 1e) in response to $\mathrm{H}-\mathrm{RasV}^{12}$ infection. In addition, knockdown of JMJD3 recovered in part the ability of cell proliferation repressed by the $\mathrm{H}-\mathrm{RasV}^{12}$, as revealed by the $\mathrm{BrdU}$ incorporation assay (Figure 1f). The representative image examples are shown in Supplementary Figure S1A. These data indicate that JMJD3 is required for SAHF formation in $\mathrm{H}$-Ras $\mathrm{V}^{12}$-induced senescence in WI38 cells.

\section{Ectopic expression of JMJD3 promoted SAHF formation.} Next, we performed gain-of-function experiments to further validate the function of JMJD3 in senescence and SAHF. We used the retrovirus construction to express JMJD3 and a JMJD3 demethylase activity domain mutant (JMJD3H1390A) in WI38 cells. The virus infection-mediated overexpression of JMJD3 was confirmed by western blotting (Figure 2a). We then showed that exogenous expression of JMJD3 induced cellular senescence (Figure 2b) and SAHF formation (Figure 2c) and reduced the number of BrdU-positive cells (Figure 2d). Image examples of the BrdU incorporation assay are presented in Supplementary Figure S1B. Meanwhile, the expression of JMJD3H1390A failed to induce cellular senescence (Figures $2 b-d$ ). We further detected the SAHF markers H3K9me3 and HMGA1 using confocal fluorescence microscopy, and the results showed a clear colocalization of DAPI foci with H3K9me3 and HMGA1 staining in cells infected with JMJD3. Contrarily, cells infected with JMJD3H1390A could not form SAHF foci (Figure 2e). Similar results were obtained in MDA-MB-231 breast cancer cells infected with JMJD3 and JMJD3H1390A (Supplementary Figure S2). Thus ectopic expression of JMJD3 is able to induce SAHF formation, and this function needs JMJD3's demethylase activity.

JMJD3 was localized to the cytoplasm in the process of SAHF formation. As a histone demethylase for $\mathrm{H} 3$ lysine27 (H3K27), JMJD3 is thought to exert its function in nucleus. Indeed, we detected apparently reduced levels of H3K27me3, H3K27me2 and H3K27me1 in nuclei during the process of $\mathrm{H}-\mathrm{RasV}^{12}$-induced SAHF formation, as revealed by western blotting and confocal fluorescence microscopy (Figure 3a,Supplementary Figure S3). Meanwhile, the level of H3K9me3 was basically unchanged. Moreover, the H3K27me3 was present as a circular ring around the DAPI foci (Supplementary Figure S4), a phenomenon also reported by Carr et al. ${ }^{28}$ We then analyzed the distribution of JMJD3 upon the formation of SAHF, and interestingly, we detected the localization of JMJD3 to the cytoplasm (Figure 3b). In addition, the JMJD3 distribution was colocalized with the Golgi protein GM130 (Supplementary Figure S5). To further confirm this result, we prepared the nuclear and cytoplasmic protein extraction, respectively, for immunoblotting, and the results indicated that the JMJD3 level in cytoplasm was much higher than that retained in nuclei (Figure $3 \mathrm{c}$ ) upon $\mathrm{H}$-Ras $\mathrm{V}^{12}$-induction. This observation inspired us to hypothesize that JMJD3 may interact with and demethylate proteins in cytoplasm, in addition to its main function of demethylating H3K27 in nuclei, during cellular senescence. This speculation will be validated in the following experiments. 
a

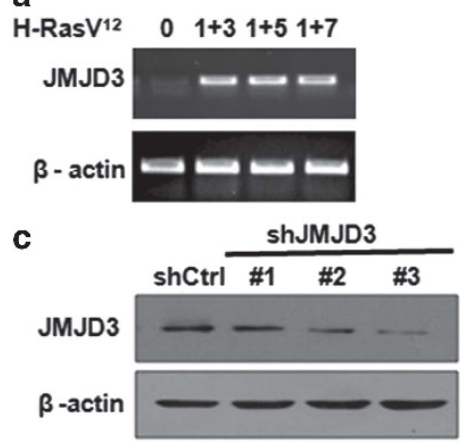

d

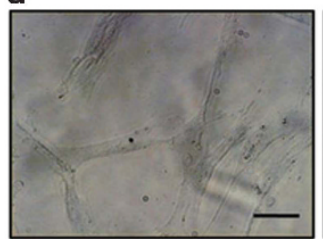

Vector

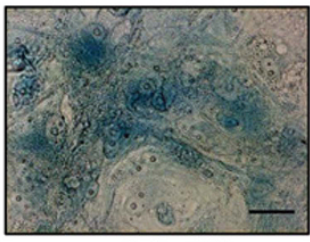

H-Ras ${ }^{12}$ b

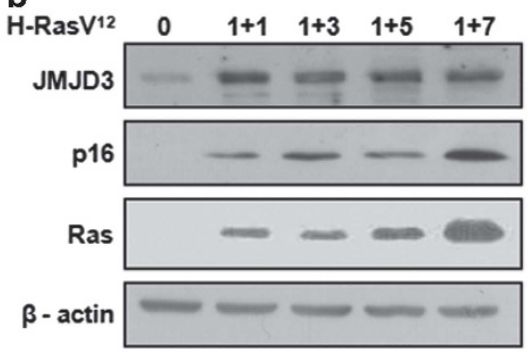

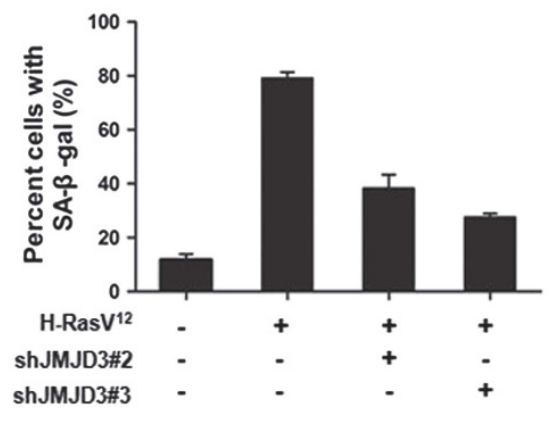
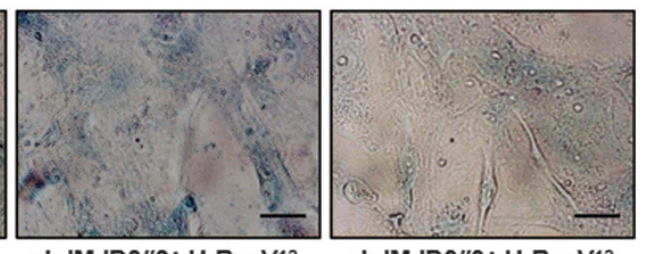

shJMJD3\#2+ H-RasV12

shJMJD3\#3+ H-RasV12
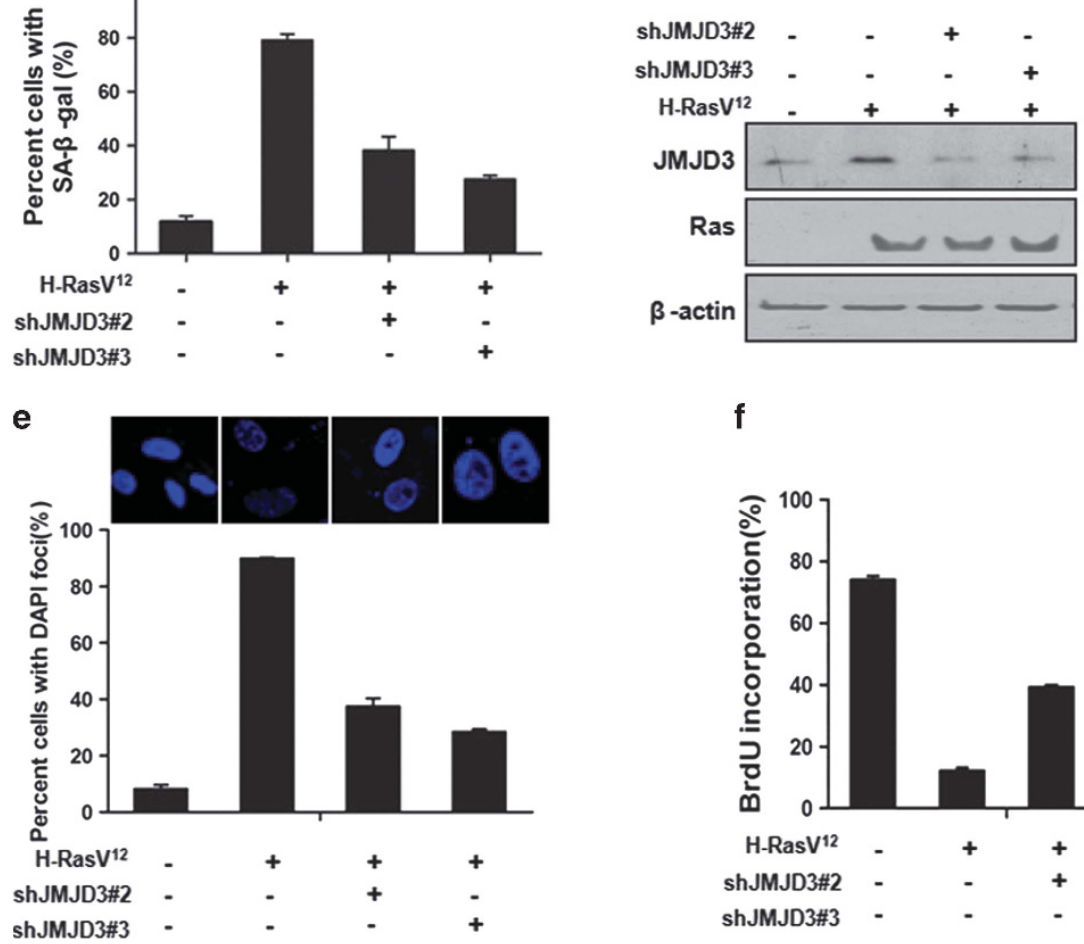

f

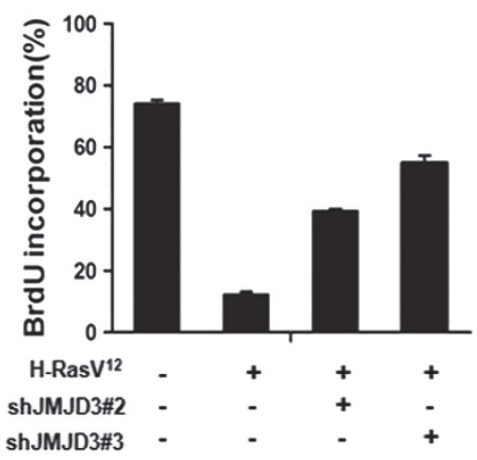

Figure 1 JMJD3 was required for Ras-induced SAHF assembly. (a and $\mathbf{b}$ ) Reverse transcriptase-PCR (a) and western blotting (b) analysis of the expression levels of JMJD3 in Ras-induced SAHF. (c) Western blots of JMJD3 protein expression in WI38 cells infected with JMJD3 shRNA (shJMJD3\#1/\#2/\#3) or the control vector shRNA (shCtr). (d) SA$\beta$-gal staining of WI38 cells. Cells were infected with shJMJD3\#2/\#3; after 2 days cells were infected with H-RasV ${ }^{12}$. The percentage of SA- $\beta$-gal positive cells was calculated (Left). Scale bars: $200 \mu \mathrm{m}$. Western blotting (Right) was performed to confirm the expression of JMJD3 and H-RasV ${ }^{12}$ after retroviral infection. (e) Cells from panel (d) were stained with 4,6-diamidino-2-phenylindole (DAPI) to calculate the percentage of cells with DAPI foci. (f) Cells from panel (d) were pulse-labeled with 5'-BrdU (bromodeoxyuridine) for $24 \mathrm{~h}$ and then immunofluorescence stained to detect the $5^{\prime}$-BrdU. Error bar represent mean \pm S.D.

JMJD3 interacted with RB through its demethylase domain. We have reported previously that the RB protein is vital to SAHF formation in cancer cells. ${ }^{23}$ In addition, methylation of RB have been shown to affect its function. ${ }^{25}$ Therefore, we intended to determine whether JMJD3 interacted with RB. First, we examined the relationship between
JMJD3 and RB by using the co-immunoprecipitation (ColP) assay, and the results showed that the ectopically expressed His-JMJD3 and Flag-RB in HEK-293T cells (Figure 4a), as well as the endogenous JMJD3 and RB in WI38 cells (Figure 4b), were co-precipitated. Furthermore, the HisJMJD3H1390A mutant was able to interact with the Flag- 
a

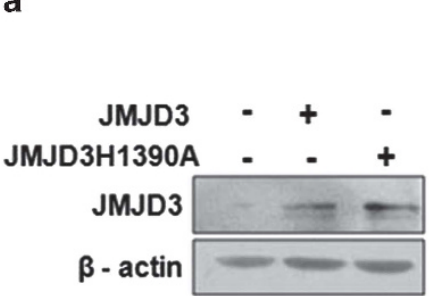

C

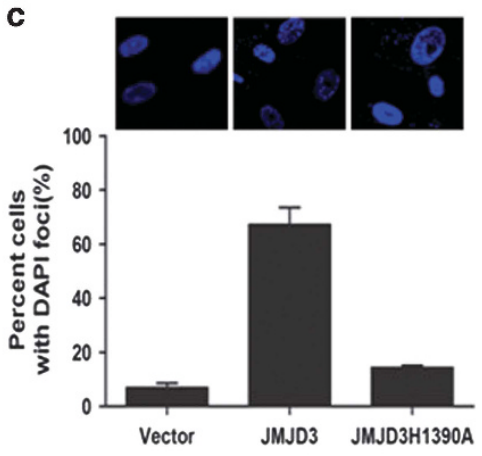

b

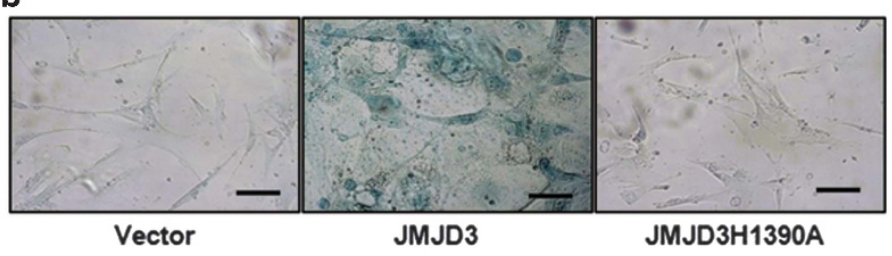

e

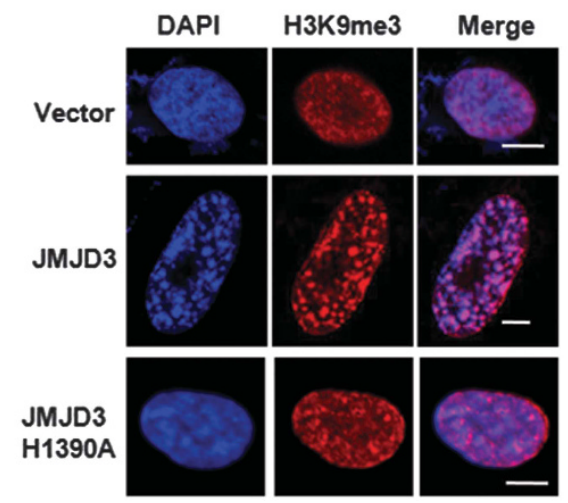

d
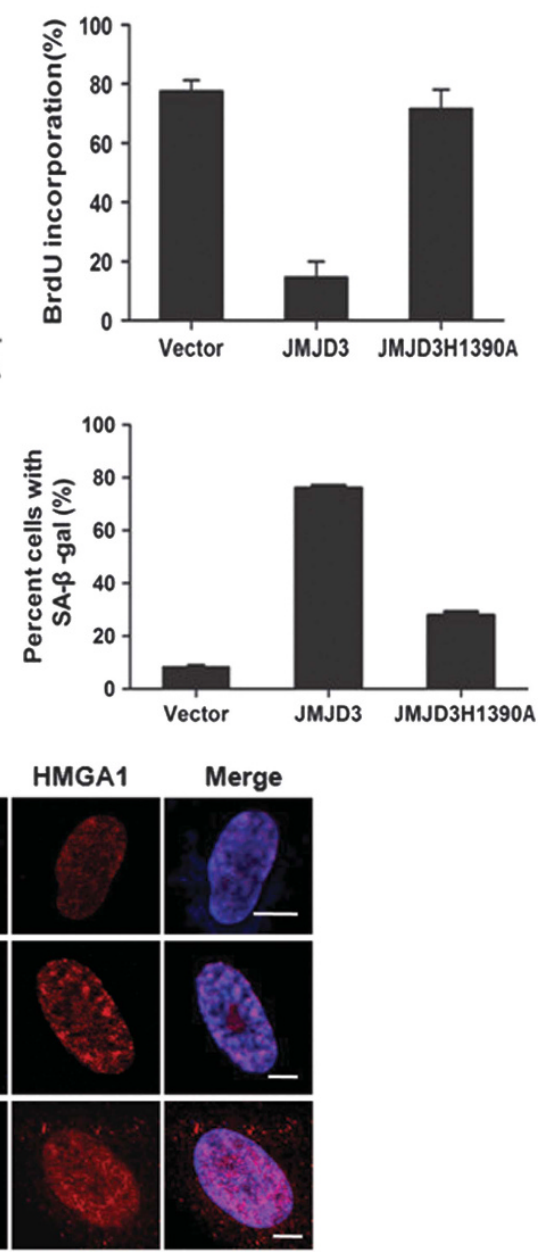

Figure 2 Ectopic JMJD3 promoted SAHF formation in WI38 cells. (a) Western blotting confirmation of the ectopic expression of JMJD3 and JMJD3H1390A after retroviral infection in WI38 cells. (b) WI38 cells were infected with JMJD3 and JMJD3H1390A for 6 days and then stained with SA- $\beta$-gal. Cells positive of SA- $\beta$-gal activity were calculated (Right). (c) WI38 cells infected with either JMJD3 or JMJD3H1390A were stained with 4,6-diamidino-2-phenylindole (DAPI), and the percentage of cells with DAPI foci was calculated. (d) WI38 cells from panel (c) were pulse-labeled with $5^{\prime}$-BrdU (bromodeoxyuridine) for $24 \mathrm{~h}$ and then immunofluorescence stained to detect the $5^{\prime}$-BrdU. A total of 100 cells were scored for $5^{\prime}$-BrdU incorporation. (e) Confocal fluorescence microscopy of SAHF markers (H3K9me3 and HMGA1) in WI38 cells and WI38 cells infected JMJD3 or JMJD3H1390A. Scale bars: $5 \mu \mathrm{m}$. Error bars represent the mean S.D. of triplicate experiments

RB as shown in Supplementary Figure S6. Next, we wanted to determine the domain of RB that directly interacted with JMJD3. Purified recombinant glutathione S-transferase (GST)-tagged RB protein from bacteria was pulled down with the cell lysate of HEK-293T cells that overexpressed JMJD3. The GST-pull-down experiments demonstrated that GST-RB, but not GST alone, interacted with JMJD3 (Figure 4c). The JmjC domain at the C-terminus has been identified as the only conserved catalytic domain of JMJD3, ${ }^{36}$ as schematically illustrated in Figure 4d. Also, as mentioned above, the demethylase activity of JMJD3 was necessary for inducing cellular senescence (Figure $2 b$ ). Therefore, we were intrigued to find out whether the JmjC was the interacting domain with RB. We purified GST-JmjC to pull down with RB, and the results indicated that the JMJD3 JmjC domain was able to interact with RB (Figure $4 e$ ).

To pinpoint the site(s) at the RB protein that interacts with JMJD3, we generated a series of truncated GST-tagged RB peptides, as indicated in Figure 4f, for pull-down experiments with JMJD3. As can be seen in Figure 4g, the fragment 659840 at the C-terminus of RB interacted with JMJD3. We then purified the Flag-RB(659-840) in HEK-293 cells and pulled down with GST-JmjC to confirm that the 659-840 domain directly bound with JmjC (Figure 4h). Together, these data demonstrate that the 659-840 domain of RB directly interacts with the demethylase domain of JMJD3.

JMJD3 demethylated RB protein at lysine810 (K810). To test whether binding of JMJD3 on RB causes the demethylation of the protein, we first examined the methylation level of $\mathrm{RB}$ in cells induced to form SAHF by $\mathrm{H}-\mathrm{RasV}^{12}$ for up to 7 days, as we showed that the RB methylation level was reduced in this process, and so does the phosphorylation of RB S807/811 (Figure 5a). Then we purified 3*Flag-RB(659840) from exponentially growing HEK-293 cells that ectopically express $3^{*}$ Flag-RB(659-840), incubated with GST-JmjC 
a

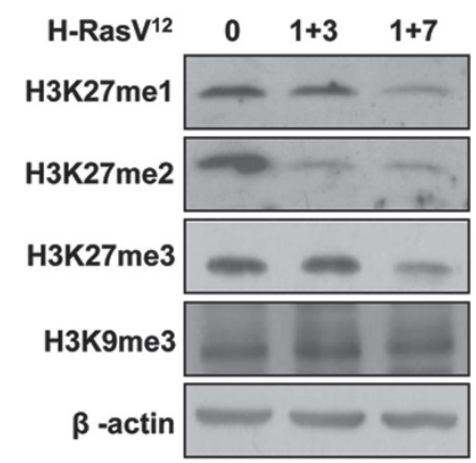

b

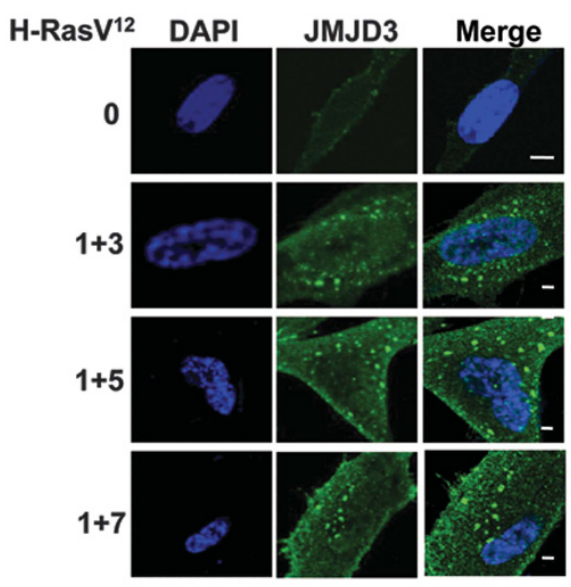

C

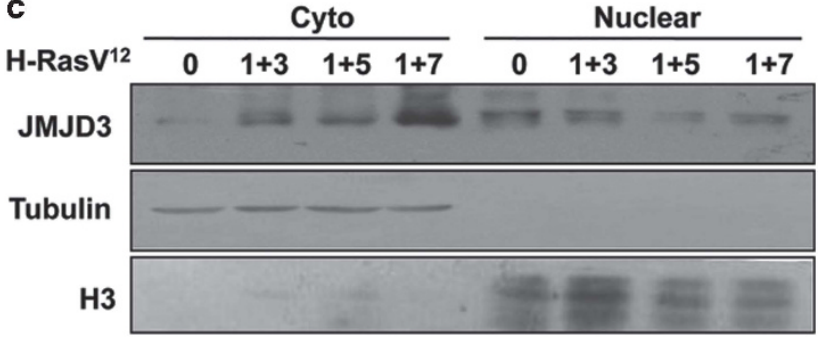

Figure 3 JMJD3 was located in cytoplasm in the process of SAHF assembly. (a) Western blots of the methylation levels of H3K27me3/me2/me1 and H3K9me3 in the process of Ras-induced SAHF formation. (b) Confocal fluorescence microscopy of JMJD3 in SAHF formation. Scale bars: $5 \mu \mathrm{m}$. (c) The presence of JMJD3 protein in cytoplasmic and nuclear extracts

in vitro and immunoblotted with an antibody recognizing methylated lysine. We found that the level of methylation of $\mathrm{RB}$ was reduced (Figure $5 \mathrm{~b}$ ), but the JmjC mut with a mutation of the demethylase activity could not demethylate $\mathrm{RB}(659-840)$ (Figure $5 \mathrm{~b}$ ), indicating that the catalytic domain of JMJD3 was able to demethylate the $\mathrm{RB}(659-840)$ fragment.

It has been reported that the K810, K860 and K873 residues of RB are methylated in vivo. ${ }^{25}$ The truncated peptide RB(659840) shown to interact with JMJD3 described above comprises the K810 residue, and this encouraged us to speculate that $\mathrm{K} 810$ may be the site JMJD3 demethylates the RB protein. Set7/9 is a methyltransferase reported to methylate the K810 and K873 sites at the C-terminal region of RB. We then performed an in vitro methylation-demethylation assay to prove this speculation. Purified Flag recombinant Set7/9 was used to methylate $\mathrm{RB}(659-840)$, and then the methylated RB (659-840) was incubated with the demethylase domain GSTJmjC and GST-JmjC mut. As a result, we detected that JmjC reverted the Set7/9-mediated methylation by using anti-lys methylation antibody (Figure 5c). Meanwhile, we performed an in vitro methylation-demethylation assay using S-adenosylL-[methyl- $\left.{ }^{3} \mathrm{H}\right]$ methionine (GE Healthcare, Buckinghamshire, UK) as a methyl donor and visualized by fluorography to further confirm the demethylation of RB by JMJD3 (Figure 5d). To provide evidence that JMJD3 demethylates the Set7/9dependent methylation of $\mathrm{RB}$ at K810, we constructed a plasmid expressing the mutant of RB(659-840K810R), purified the mutant fragment and incubated with GST-JmjC. We found that, when the K810 site was mutated in RB(659-840), the fragment could not be demethylated by JmjC (Supplementary Figure S7). This experiment manifested that only the $\mathrm{K} 810$ residue of $\mathrm{RB}(659-840)$ was demethylated by JMJD3. Next, we synthesized a peptide comprising the 18 amino acids of RB, in which the K810 was methylated (Sangon Biotech, Shanghai, China). We then showed that the methylated peptide was demethylated by JmjC but not by JmjC mut (Figure 5e).

Further we wanted to examine whether the methylation of RB K810 was depressed in WI38 cells. We infected WI38 cells with Flag-RB and Flag-RB(K810R) and then treated with or without $\mathrm{H}$-RasV ${ }^{12}$, precipitated with Flag and detected the level of methylation by an antibody recognizing the methylated lysine. The results demonstrated that the RB methylation level was decreased by H-Ras $\mathrm{V}^{12}$ (Figure 5f). When RB was mutated to $\mathrm{RB}(\mathrm{K} 810 \mathrm{R})$, the methylation level of $\mathrm{RB}$ was lower than the wild type, and the methylation of $\mathrm{RB}(\mathrm{K} 810 \mathrm{R})$ was not reduced by $\mathrm{H}-$ RasV $^{12}$ (Figure 5f). Next, we ectopically expressed a truncated mutation of RB (Flag-RB(659-840K810R)) to further confirm that the K810 of RB was demethylated during Rasinduced SAHF formation (Figure $5 \mathrm{~g}$ ). Collectively, our data demonstrate that the residue $\mathrm{K} 810$ in the $\mathrm{C}$-terminal region of RB can be demethylated by JMJD3.

Demethylation of RB K810 reduced the RB phosphorylation level. As the phosphorylation modification of RB critically influences its function, we were curious to find out whether the JMJD3-mediated RB K810 demethylation affected the RB phosphorylation to regulate the SAHF process. To prove this assumption, we assessed the phosphorylation level of RB S807/811 upon ectopic JMJD3 expression or knockdown of JMJD3 expression in WI38 cells. We showed that, when JMJD3 was overexpressed, the phosphorylation level of RB S807/811 was apparently reduced (Figure 6a). Contrarily, knockdown of JMJD3 prevented this reduction of phosphorylation level of RB S807/811 induced by H-RasV $V^{12}$ (Figure 6b). In addition, we ectopically expressed Flag-RB and Flag-RB(K810R) and detected the RB phosphorylation level, and we found that the phosphorylation of RB S807/811 was reduced when the lysine 810 was mutated to arginine (K810R); and ectopic expression of JMJD3 and H-RasV ${ }^{12}$ treatment also decreased the phosphorylation of RB (Figure 6c). These results indicated that when the RB K810 residue was demethylated (or unmethylated), the phosphorylation of RB 

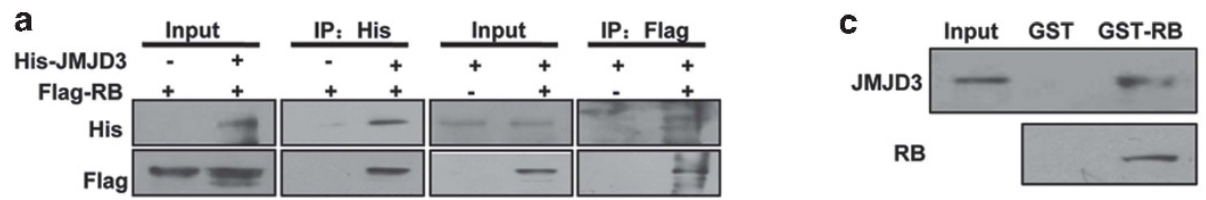

b
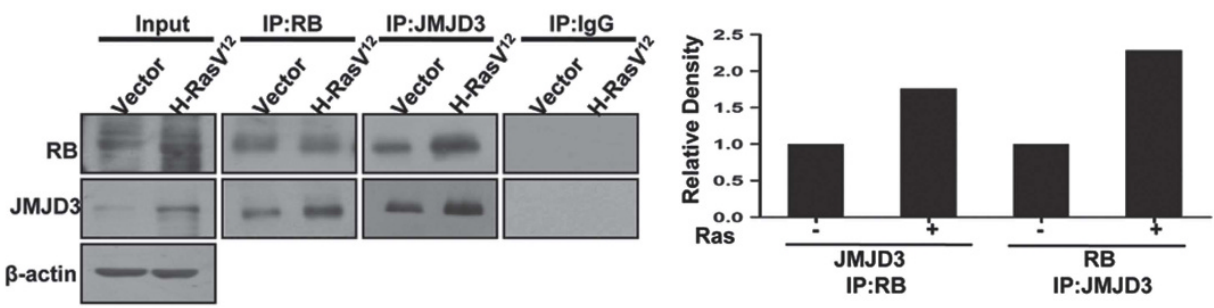

d

e
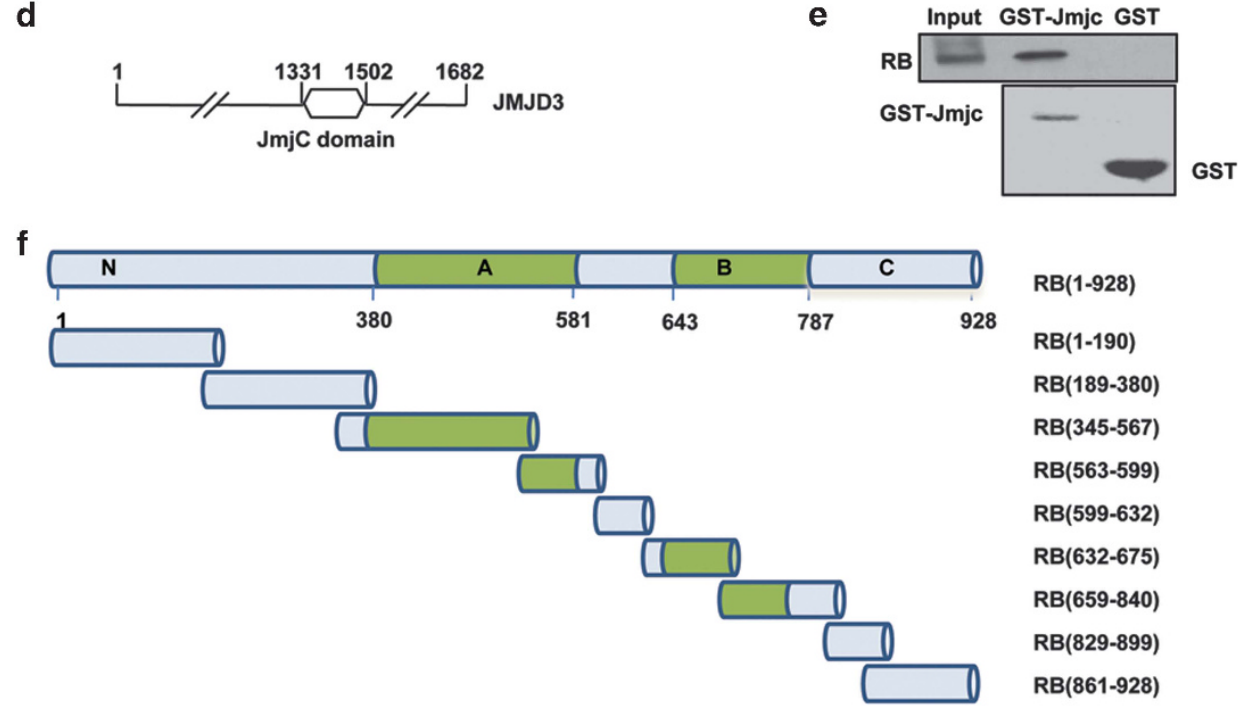

RB(1-928)

RB(1-190)

RB(189-380)

RB(345-567)

RB(563-599)

RB(599-632)

RB(632-675)

RB(659-840)

RB(829-899)

RB(861-928)

g

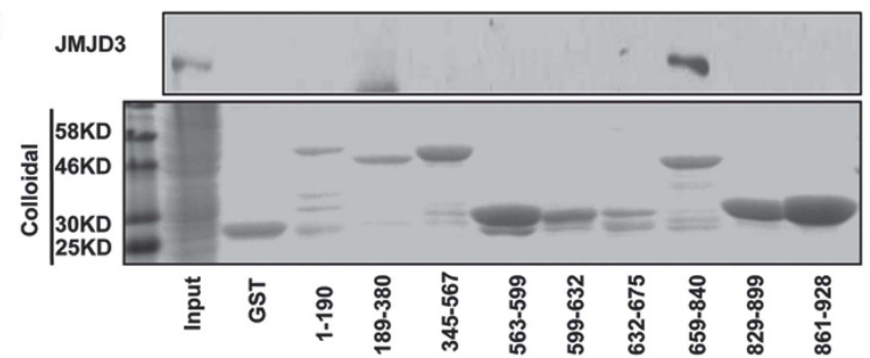

h Flag-RB(659-840)

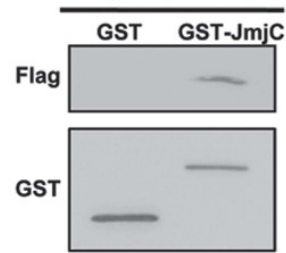

Figure 4 JMJD3 interacted with RB by its Jmjc domain. (a and $\mathbf{b}$ ) Co-IP assays showing the relationship between JMJD3 and RB. 293T cells were cotransfected with a HisJMJD3 expression vector, a Flag-RB expression vector or a mock control vector. The interaction of His-JMJD3 and Flag-RB was examined by immunoprecipitation using antiFLAG and anti-His antibodies (a). Endogenous JMJD3 and RB were coimmunoprecipitated from WI38 cells infected with H-RasV ${ }^{12}$ (b); the histogram at the right shows the data of densitometric analysis. (c) Purified GST-RB was pulled down with cell lysate of HEK-293T expressing JMJD3. (d) Schematic representation of the JmjC domain. (e) Purified GST-JmjC was pulled down with cell lysate of HEK-293 overexpressing RB. (f) Model of truncated fragments of RB. (g) Purified GST-tagged mutant RB was pulled down with JMJD3. (h) JmjC domain of JMJD3 directly interacted with RB(659-840) in vitro. GST pull-down assays was performed with the indicated GST-fused JmjC domain and purified Flag-RB(659-840) in HEK-293 cells

S807/811 was decreased, which implicated a crosstalk between the two modifications of RB protein.

To further investigate the details of the interplay between K810 methylation and $S 807 / 811$ phosphorylation of $R B$, we examined the recruitment of CDK4, a phosphorylase of RB $\mathrm{S} 807 / 811$, onto the RB protein in WI38 cells infected with JMJD3 and sh-JMJD3. The results revealed that the binding of CDK4 to RB was apparently attenuated when JMJD3 was overexpressed (Figure 6d), while this interaction was reinforced when JMJD3 was knocked down (Figure 6e).

We then explored whether mutation of the K810 residue had an impact on the expression of the E2F target genes. The realtime $\mathrm{GPCR}$ results showed that expression of wild-type RB (phosphorylated RB) was able to upregulate the E2F target genes CyclinA1, CCNA2 and CyclinB1, whereas the mutant $\mathrm{RB}(\mathrm{K} 810 \mathrm{R})$ (unphosphorylated $\mathrm{RB}$ ) could downregulate 


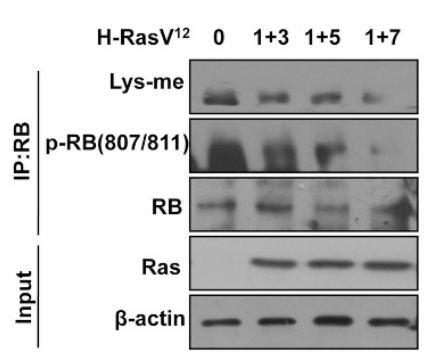

d

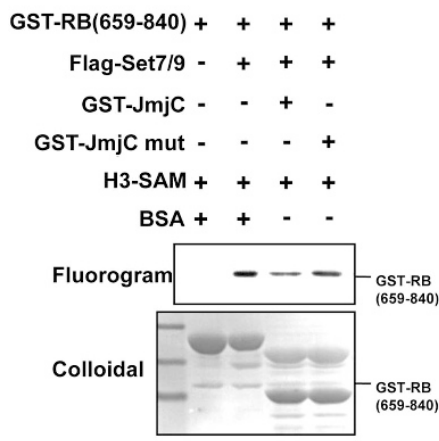

$\mathbf{f}$

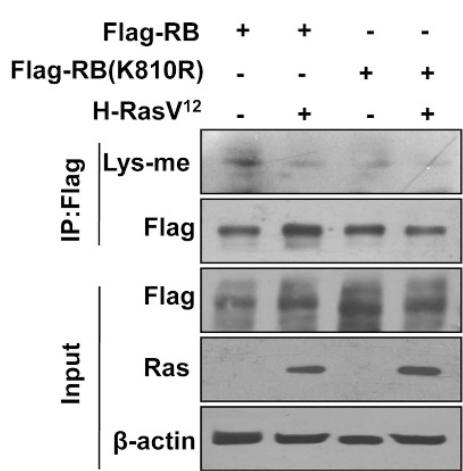

b

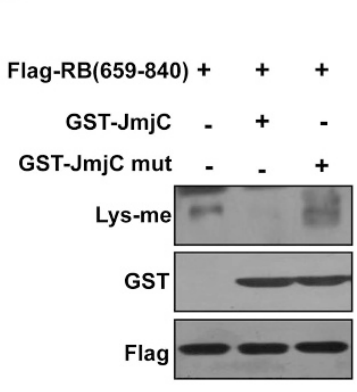

C

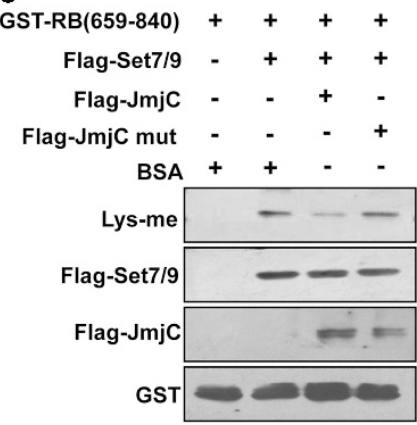

e

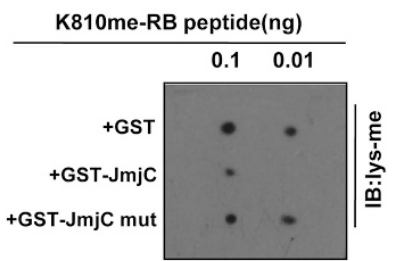

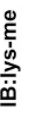

GST-RB(659-840)+

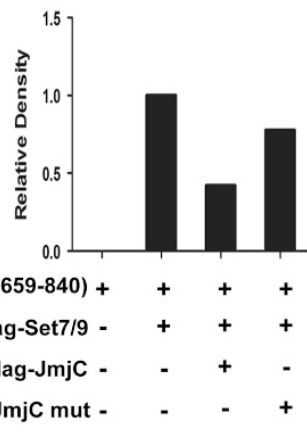

g

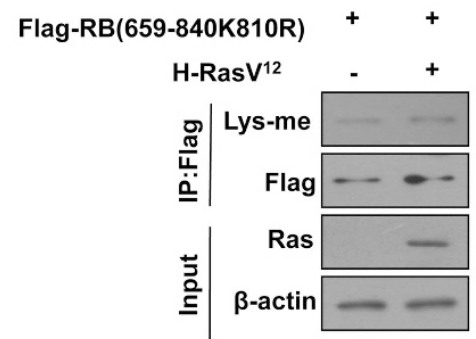

Figure 5 JMJD3 demethylated RB at K810 residue. (a) WI38 cells were infected with H-Ras $V^{12}$ for the days indicated and coimmunoprecipitated with anti-RB, and then the levels of Lys-methylation and phosphorylation of RB (Ser807/811) were determined. (b) In vitro demethylation by JmjC and JmjC mut was performed using purified Flag-RB(659840) recombinant protein from HEK-293 cells and immunoblotted with anti-Lys-me antibody. (c) In vitro methylation-demethylation of GST-fused RB(659-840). The protein was methylated by methyltransferase Set7/9 and then demethylation by JmjC or JmjC mut. The top blot shows the validation of the anti-Lys-me antibody. (d) In vitro methylationdemethylation assay. The methylated proteins were visualized with fluorography. Densitometric analysis is shown on the right. (e) In vitro demethylation assay of RB peptide containing methylated K810 was performed using JmjC or JmjC mut. Anti-Lys-me antibody was used for dot blotting. (f) WI38 cells were infected with Flag-RB/Flag-RB(K810R), then infected with or without $\mathrm{H}-$ Ras $\mathrm{V}^{12}$, coimmunoprecipitated with Flag and the level of methylated Lysine was detected. (g) WI38 cells were infected with a truncated mutation of Flag-RB (659-840K810R), then infected with or without H-RasV ${ }^{12}$, immunoprecipitated with Flag and the level of methylated Lysine was tested

MCM4, CyclinA1, CCNA2 and CyclinB1 (Figure 6f). Next, we assessed the impact of RB K810 methylation on SAHF formation; and we detected the appearance of the SAHF markers H3K9me3 and HMGA1 in WI38 cells after infecting with Flag-RB or Flag-RB(K810R). The percentage of DAPIpositive cells was increased in WI38 cells infected with Flag-RB(K810R), as compared with those cells infected with Flag-RB (Figure 6g). Confocal fluorescence microscopy revealed that cells infected with $\mathrm{RB}(\mathrm{K} 810 \mathrm{R})$ mutant formed SAHF with markers H3K9me3 and HMGA1 (Figure 6h). Collectively, these experiments provided evidence that the demethylation (or low methylation) at RB K810 was accompanied by a concomitant dephosphorylation of RB S807/811, and this resulted in the promotion of SAHF formation.

\section{Discussion}

Senescent cells retain a stable repressive state of genes that are essential for cell cycle progression. In some senescent cells, heterochromatin is condensed to form the SAHF, which suppress the transcription of genes and stabilize the senescent state of the cells. ${ }^{7}$ Elucidation of the molecular events involved in SAHF assembly provides an insight into the mechanisms of cellular senescence. Moreover, formation of 
a

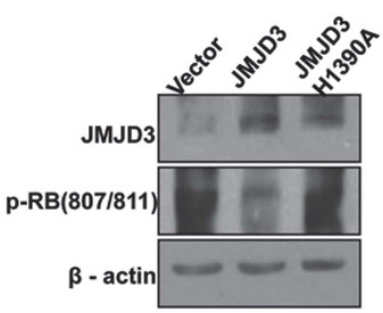

b

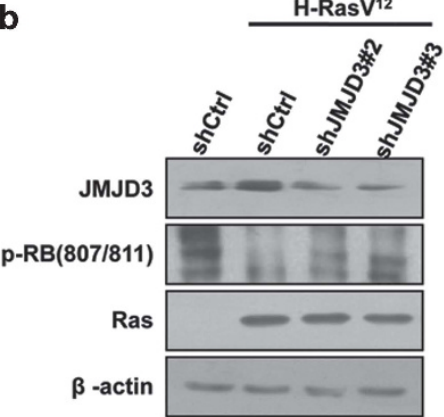

C
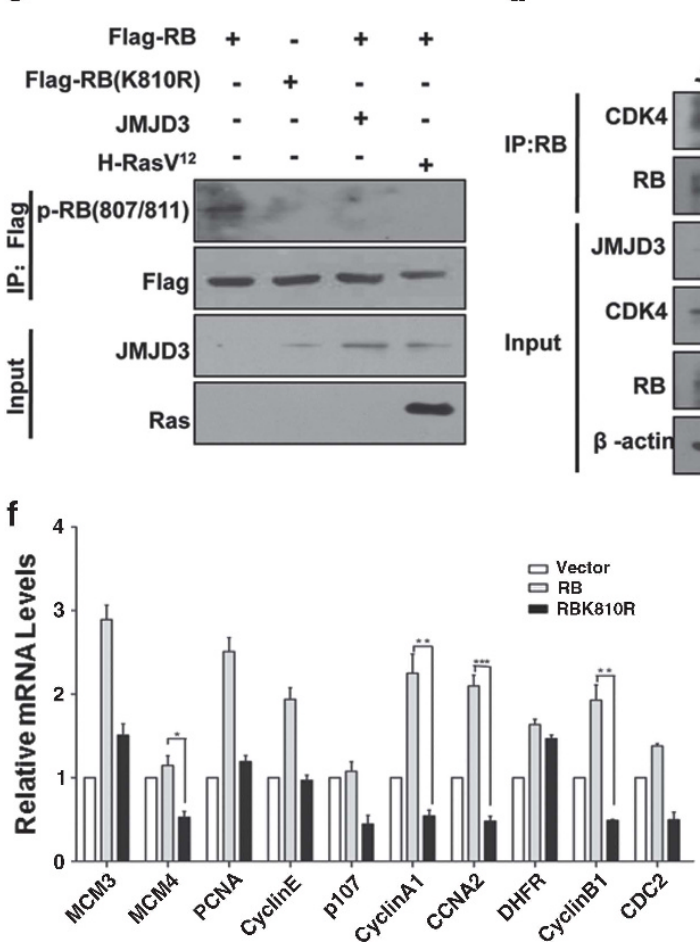

d

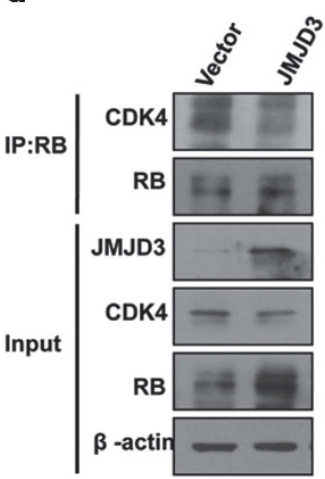

e

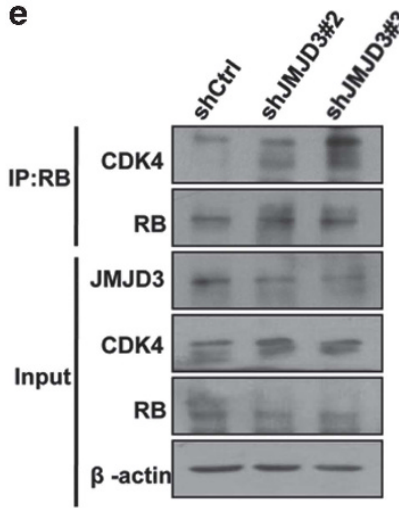

g

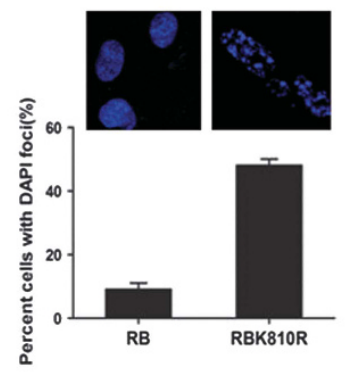

h
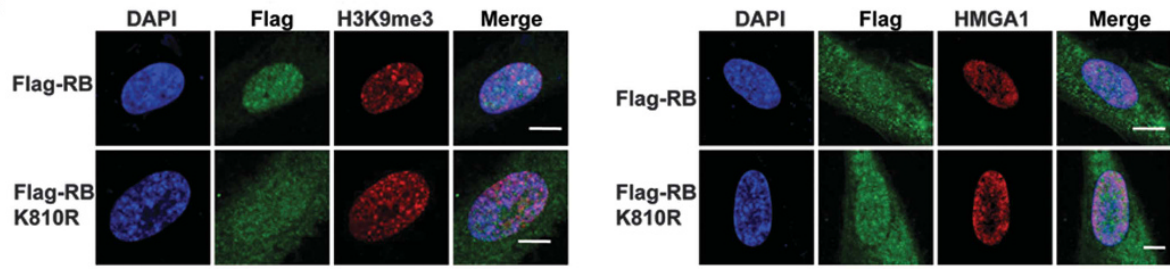

Figure 6 JMJD3-mediated RB demethylation reduced the level of RB phosphorylation. (a) Determination of phosphorylation of RB (Ser807/811) in WI38 cells ectopically expressing JMJD3 or JMJD3H1390A. (b) WI38 cells were infected with shJMJD3 to knock down endogenous JMJD3 and then infected with H-RasV ${ }^{12}$. Cell lysis was immunoblotted with anti-phospho-RB(Ser807/811) antibody. (c) WI38 cells were infected with Flag-RB, Flag-RBK810R, co-infected with Flag-RB and JMJD3 or with Flag-RB and

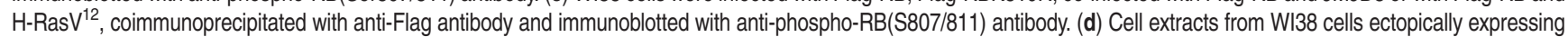
JMJD3 were coimmunoprecipitated with anti-RB antibody and immunoblotted with anti-CDK4 antibody. (e) WI38 cells were infected with shJMJD3, and the binding of CDK4 to RB was examined using co-IP. (f) mRNA expression levels of E2F targets were assessed by real-time quantitative PCR in WI38 cells ectopically expressing RB or RB(K810R). The experiments were repeated at least three times. Error bars represent mean \pm S.D. ${ }^{*} P<0.05$. (g) Cells infected with Flag-RB or Flag-RB(K810R) were stained with 4,6-diamidino2-phenylindole (DAPI) to calculate the percentage of cells with DAPI foci. (h) Immunofluorescence of Flag and H3K9me3/HMGA1 of WI38 cells infected with Falg-RB or FlagRBK810R. Scale bars: $5 \mu \mathrm{m}$

SAHF in tumor cells helps to stabilize the senescence state induced by anticancer drugs; and this has emerged as a potential therapeutic strategy with the benefit of using considerably lower doses of the drugs and hence the much reduced side effects. It is for these reasons that SAHF has attracted a great deal of research interest in recent years. In this study, we have justified a novel function of the epigenetic modifier JMJD3 to demethylate the non-histone protein RB, which has been implicated as an important player in SAHF formation. 
JMJD3 is a key histone demethylase having various roles in cellular processes, for example, it demethylates the H3K27me3 at the p16 ${ }^{\text {Ink4a }}$ promoter to activate the gene and thus to promote cellular senescence. ${ }^{31}$ Earlier studies have indicated that RB is essential for SAHF formation and for the silencing of E2F target genes. ${ }^{7}$ Our previous study demonstrates that the activated RB is also necessary for SAHF formation in tumor cells and this process is $\mathrm{p} 16$ independent. ${ }^{23}$ In a preparatory experiment of this study, we found that JMJD3 was able to induce senescence in breast cancer MDA-MB-231 cells (Supplementary Figure S2). As the MDA-MB-231 cell line is $p 16$ deficient, there must be other mechanisms than the $p 16$ pathway that JMJD3 participates in cell senescence and SAHF assembly.

Initially, we suspected that JMJD3 may work as a histone demethylase at H3K27me3 to induce SAHF formation. We examined the overall level of $\mathrm{H} 3 \mathrm{~K} 27$ methylation in senescent WI38 cells induced by $\mathrm{H}-\mathrm{RasV}^{12}$; and indeed, we detected an apparent decrease in the methylation of H3K27 (me1, me2 and me3) upon $\mathrm{H}-\mathrm{RasV}^{12}$ induction (Figure $3 \mathrm{a}$ ). Interestingly, we observed a JMJD3 accumulation in cytoplasm in the process of SAHF formation (Figure $3 b$ ). In addition, the cytoplasmic JMJD3 appeared to be distributed to the Golgi complex where many proteins are posttranslationally modified, and the JMJD3 protein was colocalized with the Golgi protein GM130 (Supplementary Figure S5). These results made us to speculate that JMJD3 may function at Golgi complex to modify non-histone proteins to participate in SAHF formation. Although JMJD3 is known to exert its function as an epigenetic modifier for histone H3K27, the possibility that it demethylates non-histones also exists. It has been reported that PRMT5 (protein arginine methyltransferase 5) can methylate the GM130 protein in Golgi complex. ${ }^{37}$

We then intended to identify the cytoplasmic proteins that potentially interact with JMJD3. Most proteins known to have function in SAHF assembly are located in nucleus, for example, Suv39H1 and HP1 $\gamma$. Nevertheless, the RB protein, a central regulator in senescence pathway, has been shown to be present both in the nucleus and in the cytoplasm ${ }^{7}$ (Figure 6h). Therefore we focused on the investigation of whether RB protein is a target of JMJD3 in the cytoplasm in SAHF formation.

The $\mathrm{RB} / \mathrm{E} 2 \mathrm{~F}$ pathway is vital for tumor suppression. It is well known that, in early $\mathrm{G} 1$ phase, $\mathrm{RB}$ is phosphorylated at $\mathrm{S} 807 / 811$ by CyclinD/CDK4/6, and the phosphorylated RB is released from E2F to activate genes required for cells to enter into $S$ phase, while in senescent cells the RB S807/811 are dephosphorylated. ${ }^{18,38,39}$ Inspired by the recent discovery that the posttranslational modifications of $R B$, such as phosphorylation, methylation and acetylation, may affect the RB function in regulating cellular activities, we were interested in investigation of the possible crosstalks of different modifications of RB in SAHF formation. A major finding of this study is that demethylation of RB also impacts SAHF formation and that JMJD3 acts as a cofactor in RB/E2F pathway. Significantly, we have provided evidence that the JMJD3-mediated demethylation of RB K810 impairs the phosphorylation of RB by suppressing the binding of CDK4 to $R B$, and this reduces the level of RB phosphorylation at S807/811 (Figure 6d). Furthermore, the hypophosphorylated RB resulted from this crosstalk of modifications potentiates the interaction between $\mathrm{RB}$ and E2F, which in turn represses the expression of E2F target genes, such as MCM4, CyclinA1, CCNA2 and CyclinB1 (Figure 6f). Interestingly, other E2F target genes, including MCM3, PCNA and DHFR, were not reduced by RB(K810R) mutant in contrast to the vector (Figure 6f). Presumably, repression of CyclinA1, CCNA2 and CyclinB1 genes finally blocks the cell cycle progression and induces cellular senescence and SAHF formation in WI38 cells (Figure 6h). This has been the first piece of evidence that the crosstalk between methylation/demethylation and phosphorylation of $\mathrm{RB}$ protein at specific residues regulates its function in SAHF formation.

Earlier studies have demonstrated that methylation at RB K810 by Set7/9 retains the hypophosphorylated state of RB and suppresses cellular growth in U2OS cells, ${ }^{28}$ whereas the SMYD2-dependent methylation of RB K810 promotes cell cycle progression in SW780 and RT4 cells. ${ }^{29}$ Apparently, our results are in favor of that described by Cho et al. ${ }^{29}$ that methylation of RB K810 promotes cell cycle progression.

To summarize, we have proposed a working model to depict the JMJD3 function in regulation of cellular senescence and SAHF assembly, based on data arising from this study, as well as on other relevant information (Figure 7). Briefly, this model illustrates that in normal growing cells $\mathrm{RB}$ is phosphorylated by CDK4, resulting in the release of the transcription factor E2F and hence the activation of the transcription of its target genes. This finally promotes the cell cycle progression and proliferation (Figure 7, upper). On the other hand, when cells are exposed to the senescence signals, for example, the oxidative stress, DNA damage or hyperoncogenic pressure and so on, JMJD3 demethylates RB at K810, preventing CDK4 from binding. This reduces the level of phosphorylation of $\mathrm{RB}$ at S807/811 and induces senescence and SAHF formation (Figure 7, lower). Overall, this study justifies the novel function of the histone demethylase JMJD3 as a promoting factor of the SAHF formation in senescent WI38 cells, through influencing the RB/E2F pathway. These findings provide new insights into the mechanisms of cellular senescence and SAHF assembly.

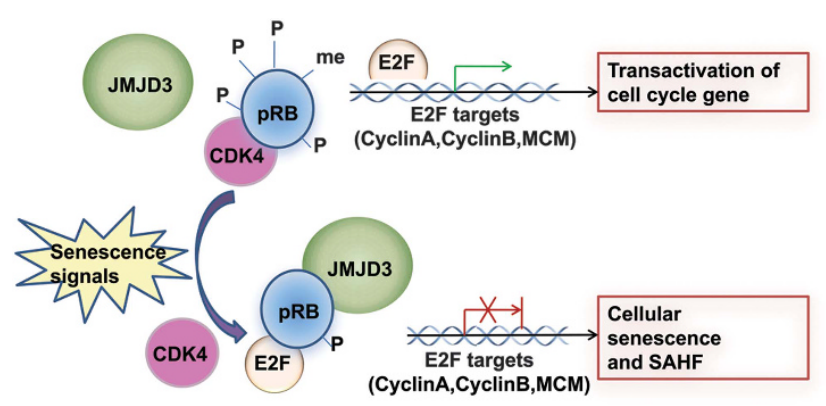

Figure 7 A proposed working model for JMJD3 function in regulation of cellular senescence. In normal growing cells, RB is phosphorylated by CDK4, releases E2F, promotes the transcription of its target genes and results in cell cycle progression. When cells are exposed to the senescence signals, such as oxidative stress, DNA damage or hyperoncogenic signaling pressure, JMJD3 demethylates RB at K810, represses CDK4 binding to $\mathrm{RB}$, reduces the level of phosphorylation of $\mathrm{RB}$ at S807/811 and induces senescence and SAHF formation 


\section{Materials and Methods}

Cell culture and antibodies. All the cell lines (WI38, HEK-293T, HEK-293) were obtained from the ATCC (Manassas, VA, USA) and maintained according to the protocols provided by the ATCC. WI38 cells were cultured in MEM medium (Gibco, Grand Island, NY, USA) supplemented with 10\% HyClone (Thermo, Rockford, IL, USA), $1 \mathrm{mM}$ sodium pyruvate and $1500 \mathrm{mg} / \mathrm{l}$ sodium bicarbonate. HEK-293T and HEK-293 cells were cultured in DMEM medium (Sigma, St. Louis, MO, USA) containing 10\% FBS (ExCell Bio, Shanghai, China).

The following antibodies were used: anti-JMJD3, anti-methylated lysine (Abcam, Hong Kong, China), anti-p-RB (807/811) (Cell Signal Technology, Danvers, MA, USA), anti-Ras (Upstate, Billerica, MA, USA), anti-H3K27me3, anti-H3K27me2, antiH3K27me1, anti-H3 (Millipore, Billerica, MA, USA), anti-RB, anti-p16 (BD Biosciences, San Jose, CA, USA), anti-His, anti-GST (Sungene Biotech, Tianjin, China), anti-Flag (Abcam), anti- $\beta$-actin (Sigma), anti-CDK4 (Santa Cruz, Dallas, TX, USA), and anti-Flag mouse monoclonal M2 (Sigma).

Plasmids. The MSCV-JMJD3 and MSCV-JMJD3 H1390A retroviral expression plasmids were bought from Addgene (Cambridge, MA, USA). The His-JMJD3 expression plasmids and the bacterial expression plasmid pGEX-4T1-JmjC were gifts from Dr. Charlie Degui Chen (State Key Laboratory of Molecular Biology, Institute of Biochemistry and Cell Biology, Shanghai Institutes for Biological Sciences, Chinese Academy of Sciences). The JMJD3 cDNA (3991-4506) was generated by PCR to construct GST-JmjC domain of JMJD3. The lentiviral expression plasmid Pwpxld-Flag-RB and Pwpxld-Flag-RB(K810R) were constructed by inserting a PCR amplification fragment into the Pwpxld vector. For lentiviralmediated gene silencing, the shJMJD3\#1, shJMJD3\#2 and shJMJD3\#3 oligonucleotides were designed to target human genes and were cloned into the pDSL-hpUGIP vector. The sequences of the shRNAs are listed as follows.

ShCtrl: 5'-GATCCCCTTCTCCGAACGTGTCACGTTTCAAGAGAACGTGACACG TTCGGAGAATTTTTC-3' and 5'-TCGAGAAAAATTCTCCGAACGTGTCACGTTCT CTTGAAACGTGACACGTTCGGAGAAGGG-3';

shJMJD3\#1: 5'-GATCCCCGCATCTATCTGGAGAGCAATTCAAGAGATTGCTCT CCAGATAGATGCTTTTTC-3' and 5'-TCGAGAAAAAGCATCTATCTGGAGAGCAA TCTCTTGAATTGCTCTCCAGATAGATGCGGG-3';

shJMJD3\#2: 5'-GATCCCCGGAATGAGGTGAAGAACGTTTCAAGAGAACGTTC TTCACCTCATTCCTTTTTC- $3^{\prime}$ and 5'-TCGAGAAAAAGGAATGAGGTGAAGAACG TTCTCTTGAAACGTTCTTCACCTCATTCCGGG-3';

shJMJD3\#3: 5'-GATCCCCGCAAGTGTGGAACTTGCTACATTCAAGAGATGTAG CAAGTTCCACACTTGCTTTTTC- ${ }^{\prime}$ and $5^{\prime}$-TCGAGAAAAAGCAAGTGTGGAACTT GCTACATCTCTTGAATGTAGCAAGTTCCACACTTGCGGG-3'.

The bacterial expression plasmid pGEX-4T1-RB and the other truncated mutation were gifts from Dr. Julien Sage (the Institute for Stem Cell Biology and Regenerative Medicine, Stanford University, Stanford, CA, USA). 3*flag-pCMV-RB (659-840) and $3^{*}$ flag-pCMV-RB $(659-840) K 810 R$ were constructed from the pGEX-4T1-RB (659-840) expression vector by inserting a PCR amplification fragment.

DAPI staining. For SAHF detection, cells were plated on cover slips. After infected to express H-RasV ${ }^{12}$ for the indicated days, cells were fixed in $1 \%$ formaldehyde in PBS for 10 min at $37^{\circ} \mathrm{C}$ and permeabilized with $0.2 \%$ Triton X-100 in PBS for 10 min at room temperature. Cells were then washed three times in PBS and incubated with $500 \mathrm{nM}$ DAPI (Invitrogen, Paisley, UK). Cells were documented using a charge-coupled device camera (Canon, Tokyo, Japan) attached to a microscope (OLYMPUS, Tokyo, Japan).

Cellular immunofluorescence. Cells were fixed in $1 \%$ formaldehyde in culture medium for $10 \mathrm{~min}$ at $37^{\circ} \mathrm{C}$ and permeabilized with $0.2 \%$ Triton X-100 in PBS for $10 \mathrm{~min}$ at room temperature. Cells were washed twice in PBS and blocked for $1 \mathrm{~h}$ with $5 \% \mathrm{BSA}$ in PBS and then incubated with primary antibodies for $1 \mathrm{~h}$ at room temperature, washed three times in PBS and incubated with secondary antibodies for $50 \mathrm{~min}$ at room temperature. Photographs were taken using a confocal microscope (OLYMPUS). The primary antibodies were rabbit antiH3K9me3 (Millipore), rabbit anti-JMJD3 (Abcam), rabbit anti-H3K27me3 (Millipore) and mouse anti-HMGA1 (Santa Cruz).

Detection of SA- $\beta$-gal. Cells were fixed in $1 \%$ formaldehyde, washed three times with PBS (pH 6.0) containing $1 \mathrm{mM} \mathrm{MgCl}$ and exposed overnight at $37^{\circ} \mathrm{C}$ to a solution containing $1 \mathrm{mg} / \mathrm{ml}$ 5-bromo-4-chloro-3-indolyl- $\beta$-D-galactopyranoside, $0.12 \mathrm{mM}$ potassium ferrocyanide, $0.12 \mathrm{mM}$ potassium ferricyanide and $1 \mathrm{mM} \mathrm{MgCl}_{2}$ PBS buffer ( $\mathrm{pH}$ 6.0). Cells were observed under a fluorescence microscope
(OLYMPUS). All the experiments were repeated three times, and one of the representative results is shown.

BrdU incorporation assay. For the DNA synthesis assay, BrdU (BD Pharmingen, BD Biosciences) was added to the medium $(10 \mathrm{nmol} / \mathrm{ml})$ at the indicated time points, and cells were cultured for an additional $24 \mathrm{~h}$. Cells were then fixed, permeabilized and denatured. BrdU incorporation was measured by immunofluorescence using an anti-BrdU serum (BD Pharmingen), and the nuclei were stained with DAPI. The stained cells were visualized under a fluorescence microscope, and at least 200 cells were scored for BrdU incorporation. Each experiment was repeated at least three times, and the results are displayed as a percentage of BrdU-positive cells in histogram.

Co-IP assay. Total cell extraction was precleared with salmon sperm DNA/ protein A/G-agarose beads (Millipore). Mouse anti-Flag and mouse anti-His was added for immunoprecipitation. The precipitates were then subjected to SDS/PAGE followed by transfer onto a PVDF membrane and incubation with anti-His or Flag serum. Samples were detected using the Super Signal West Pico Chemiluminescent Substrate (Pierce, Rockford, IL, USA) detection method in accordance with the manufacturer's instructions.

GST pull-down assay. GST or GST fusion proteins were expressed in bacteria induced with isopropyl- $\beta$-D-thio-galactoside and purified. Equal amounts of GST or GST fusion proteins were incubated with glutathione-Sepharose 4B beads (GE Healthcare) and then washed three times with TEN buffer (20-mM Tris at $\mathrm{pH}$ 7.4, $0.1 \mathrm{mM}$ EDTA and $100 \mathrm{mM} \mathrm{NaCl}){ }^{40}$ Flag fusion proteins purified from HEK-293T cells was incubated with GST or GST fusion proteins. The beads were washed three times with TENT buffer $(0.5 \%$ Nonidet P-40, $20 \mathrm{mM}$ Tris at pH7.4, $0.1 \mathrm{mM}$ EDTA and $300 \mathrm{mM} \mathrm{NaCl}$ ) and analyzed by western blotting with anti-Flag or anti-GST antibody.

In vitro methylation and demethylation assay. To perform in vitro methylation assay, purified Flag-Set7/9 in HEK-293T cells were incubated with recombinant GST-RB (residues 659-840) as a substrate and $2 \mathrm{mCi}$ S-adenosyl-L[methyl-3H] methionine (GE Healthcare) as a methyl donor in a mixture of $10 \mathrm{ml}$ of methylase activity buffer ( $20 \mathrm{mmo} / / \mathrm{Tris}-\mathrm{HCl}, 4 \mathrm{mmol} / \mathrm{IEDTA}$, at $\mathrm{pH} 8.0)$, for $1 \mathrm{~h}$ at $30^{\circ} \mathrm{C}$. After methylation, $40 \mathrm{nmol} 5$ '-deoxy-5'-(methylthio) adenosine (Sigma) was added to stop the methylase activity. The substrate was incubated with the purified fusion protein of GST-JmjC domain of JMJD3 in demethylation buffer $(20 \mathrm{mM}$ Tris$\mathrm{HCl} \mathrm{pH} \mathrm{7.3,} 150 \mathrm{mM} \mathrm{NaCl}, 50 \mu \mathrm{M}\left(\mathrm{NH}_{4}\right) 2 \mathrm{Fe}\left(\mathrm{SO}_{4}\right) 2+6\left(\mathrm{H}_{2} \mathrm{O}\right), 1 \mathrm{mM} \alpha$-ketoglutarate and $2 \mathrm{mM}$ ascorbic acid) for $4 \mathrm{~h}$ at $37^{\circ} \mathrm{C} . .^{36,41}$ Substrate was reacted in a total volume of $40 \mu \mathrm{l}$ of reaction mixture. The reaction was stopped with SDS loading buffer, and western blotting analysis was performed. The sequence of the synthetized K810 methylated peptide (Sangon Biotech) is GGNIYISPLK(monomethy)SPYKISEG.

Cytoplasmic and nuclear extracts. Cells were collected, gently resuspended in $500 \mu \mathrm{l}$ cytoplasmic extract buffer $(10 \mathrm{mM}$ HEPES, $60 \mathrm{mM} \mathrm{KCl}$, $1 \mathrm{mM}$ EDTA, $0.075 \%$ (v/v) NP40, $1 \mathrm{mM}$ DTT and $1 \mathrm{mM}$ PMSF, adjusted to $\mathrm{pH} 7.6$ ) and incubated on ice for $30 \mathrm{~min}$. Cells were then centrifuged at 14000 r.p.m. for $1 \mathrm{~min}$ at $4^{\circ} \mathrm{C}$, and the supernatant was transferred and saved. The nuclear pellets were resuspended in 100-200 $\mu$ nuclear extract buffer $(20 \mathrm{mM}$ Tris Cl, $420 \mathrm{mM}$ $\mathrm{NaCl}, 1.5 \mathrm{mM} \mathrm{MgCl}_{2}, 0.2 \mathrm{mM}$ EDTA, $1 \mathrm{mM}$ PMSF and 25\% (v/v) glycerol, adjusted to $\mathrm{pH} 8.0)$ for $30 \mathrm{~min}$ on ice with vortexing. The extracts were centrifuged at 14000 r.p.m. for $15 \mathrm{~min}$ at $4^{\circ} \mathrm{C}$, and the supernatant (nuclear fraction) was transferred to a clean microcentrifuge tube.

Statistics. Data are presented as mean \pm S.D. as indicated. Student's $t$-test (two-tailed) was used to determine statistic significance of differences between groups. $P<0.05$ was considered statistically significant. All statistical analyses were carried out using the SPSS 17.0 statistical software (SPSS software, Chicago, IL, USA).

\section{Conflict of Interest}

The authors declare no conflict of interest. 
Acknowledgements. We thank Dr. Charlie Degui Chen and Dr. Julien Sage for providing the plasmids mentioned in Methods section. This work was supported by grants from the National Natural Science Foundation of China (grant numbers $31100998,31371294,31170719$ ), and a grant from the Northeast Normal University, China (12SSXT137).

1. Campisi J, d'Adda di Fagagna F. Cellular senescence: when bad things happen to good cells. Nat Rev Mol Cell Biol 2007; 8: 729-740.

2. Salama R, Sadaie M, Hoare M, Narita M. Cellular senescence and its effector programs. Genes Dev 2014; 28: 99-114.

3. Ben-Porath I, Weinberg RA. The signals and pathways activating cellular senescence. Int J Biochem Cell Biol 2005; 37: 961-976.

4. Serrano M, Lin AW, McCurrach ME, Beach D, Lowe SW. Oncogenic ras provokes premature cell senescence associated with accumulation of p53 and p16INK4a. Cell 1997; 88: 593-602.

5. Hemann MT, Narita M. Oncogenes and senescence: breaking down in the fast lane. Genes Dev 2007; 21: 1-5.

6. Longhese MP. DNA damage response at functional and dysfunctional telomeres. Genes Dev 2008; 22: 125-140.

7. Narita $M$, Nuñez $S$, Heard $E$, Narita $M$, Lin AW, Hearn $S A$ et al. Rb-mediated heterochromatin formation and silencing of E2F target genes during cellular senescence. Cell 2003; 113: 703-716.

8. Schulz L, Tyler J. Heterochromatin focuses on senescence. Mol Cell 2005; 17: 168-170.

9. Narita M, Narita M, Krizhanovsky V, Nunez S, Chicas A, Hearn SA et al. A novel role for highmobility group a proteins in cellular senescence and heterochromatin formation. Cell 2006; 126: $503-514$.

10. Zhang R, Poustovoitov MV, Ye X, Santos HA, Chen W, Daganzo SM et al. Formation of MacroH2A-containing senescence-associated heterochromatin foci and senescence driven by ASF1a and HIRA. Dev Cell 2005; 8: 19-30.

11. Zhang R, Chen W, Adams PD. Molecular dissection of formation of senescence-associated heterochromatin foci. Mol Cell Biol 2007; 27: 2343-2358.

12. Chandra T, Kirschner K, Thuret JY, Pope BD, Ryba T, Newman S et al. Independence of repressive histone marks and chromatin compaction during senescent heterochromatic layer formation. Mol Cell 2012; 47: 203-214.

13. Sadaie M, Salama R, Carroll T, Tomimatsu K, Chandra T, Young AR et al. Redistribution of the Lamin B1 genomic binding profile affects rearrangement of heterochromatic domains and SAHF formation during senescence. Genes Dev 2013; 27: 1800-1808.

14. Shah PP, Donahue G, Otte GL, Capell BC, Nelson DM, Cao K et al. Lamin B1 depletion in senescent cells triggers large-scale changes in gene expression and the chromatin landscape. Genes Dev 2013; 27: 1787-1799.

15. Zhang R, Adams PD. Heterochromatin and its relationship to cell senescence and cancer therapy. Cell Cycle 2007; 6: 784-789.

16. Gonzalo S, Garcia-Cao M, Fraga MF, Schotta G, Peters AH, Cotter SE et al. Role of the RB1 family in stabilizing histone methylation at constitutive heterochromatin. Nat Cell Biol 2005; 7: $420-428$.

17. Kaelin WG Jr.. Functions of the retinoblastoma protein. BioEssays 1999; 21: 950-958.

18. Uchida C. The retinoblastoma protein: functions beyond the G1-S regulator. Curr Drug Targets 2012; 13: 1622-1632.

19. Dick FA, Dyson N. pRB contains an E2F1-specific binding domain that allows E2F1-induced apoptosis to be regulated separately from other E2F activities. Mol Cell 2003; 12: 639-649.

20. Burke JR, Hura GL, Rubin SM. Structures of inactive retinoblastoma protein reveal multiple mechanisms for cell cycle control. Genes Dev 2012; 26: 1156-1166.

21. Ye X, Zerlanko B, Zhang R, Somaiah N, Lipinski M, Salomoni P et al. Definition of pRB- and p53-dependent and -independent steps in HIRA/ASF1a-mediated formation of senescenceassociated heterochromatin foci. Mol Cell Biol 2007; 27: 2452-2465.
22. Nielsen SJ, Schneider R, Bauer UM, Bannister AJ, Morrison A, O'Carroll D et al. Rb targets histone H3 methylation and HP1 to promoters. Nature 2001; 412: 561-565.

23. Zhang Y, Gao Y, Zhao L, Han L, Lu Y, Hou P et al. Mitogen-activated protein kinase $\mathrm{p} 38$ and retinoblastoma protein signalling is required for DNA damage-mediated formation of senescence-associated heterochromatic foci in tumour cells. FEBS J 2013; 280: 4625-4639.

24. Longworth MS, Dyson NJ. pRb a local chromatin organizer with global possibilities. Chromosoma 2010; 119: 1-11.

25. Munro S, Carr SM, La Thangue NB. Diversity within the pRb pathway: is there a code of conduct? Oncogene 2012; 31: 4343-4352.

26. Munro S, Khaire N, Inche A, Carr S, La Thangue NB. Lysine methylation regulates the pRb tumour suppressor protein. Oncogene 2010; 29: 2357-2367.

27. Saddic LA, West LE, Aslanian A, Yates JR, Rubin SM, Gozani $O$ et al. Methylation of the retinoblastoma tumor suppressor by SMYD2. J Biol Chem 2010; 285: 37733-37740.

28. Carr SM, Munro S, Kessler B, Oppermann U, La Thangue NB. Interplay between lysine methylation and Cdk phosphorylation in growth control by the retinoblastoma protein. EMBO J 2011; 30: 317-327.

29. Cho HS, Hayami S, Toyokawa G, Maejima K, Yamane Y, Suzuki T et al. RB1 methyl by SMYD2 enhances cell cycle progression through an increase of RB1 phosphorylation1. Neoplasia 2012; 14: 476-486.

30. Agger K, Cloos PA, Christensen J, Pasini D, Rose S, Rappsilber J et al. UTX and JMJD3 are histone H3K27 demethylases involved in HOX gene regulation and development. Nature 2007; 449: 731-734.

31. Barradas M, Anderton E, Acosta JC, Li S, Banito A, Rodriguez-Niedenfuhr M et al. Histone demethylase JMJD3 contributes to epigenetic control of INK4a/ARF by oncogenic RAS. Genes Dev 2009; 23: 1177-1182.

32. Agger K, Cloos PA, Rudkjaer L, Williams K, Andersen G, Christensen J et al. The H3K27me3 demethylase JMJD3 contributes to the activation of the INK4A-ARF locus in response to oncogene- and stress-induced senescence. Genes Dev 2009; 23: 1171-1176.

33. Ene Cl, Edwards L, Riddick G, Baysan M, Woolard K, Kotliarova S et al. Histone demethylase Jumonji D3 (JMJD3) as a tumor suppressor by regulating p53 protein nuclear stabilization. PLoS One 2012; 7: e51407.

34. Sola S, Xavier JM, Santos DM, Aranha MM, Morgado AL, Jepsen K et al. p53 interaction with JMJD3 results in its nuclear distribution during mouse neural stem cell differentiation. PLOS One 2011; 6: e18421.

35. Pfau R, Tzatsos A, Kampranis SC, Serebrennikova OB, Bear SE, Tsichlis PN. Members of a family of JmjC domain-containing oncoproteins immortalize embryonic fibroblasts via a JmjC domain-dependent process. Proc Natl Acad Sci USA 2008; 105: 1907-1912.

36. Xiang $Y$, Zhu ZQ, Han G, Lin HQ, Xu LY, Chen CD. JMJD3 is a histone H3K27 demethylase. Cell Res 2007; 17: 850-857.

37. Zhou ZW, Sun XT, Zou ZH, Sun LT, Zhang T, Guo SS et al. PRMT5 regulates Golgi apparatus structure through methylation of the golgin GM130. Cell Res 2010; 20: 1023-1033.

38. Schaal C, Pillai S, Chellappan SP. The Rb-E2F transcriptional regulatory pathway in tumor angiogenesis and metastasis. Adv Cancer Res 2014; 121: 147-182.

39. Sun Y, Lou XM, Yang M, Yuan CF, Ma L, Xie BK et al. Cyclin-dependent kinase 4 may be expressed as multiple proteins and have functions that are independent of binding to CCND and $\mathrm{RB}$ and occur at the $\mathrm{S}$ and $\mathrm{G}(2) / \mathrm{M}$ phases of the cell cycle. Cell Cycle 2013; 12: $3512-3525$.

40. Huang J, Sengupta R, Espejo AB, Lee MG, Dorsey JA, Richter M et al. p53 is regulated by the lysine demethylase LSD1. Nature 2007; 449: 105-U180.

41. Cho HS, Suzuki T, Dohmae N, Hayami S, Unoki M, Yoshimatsu M et al. Demethylation of RB regulator MYPT1 by histone demethylase LSD1 promotes cell cycle progression in cancer cells. Cancer Res 2011; 71: 655-660.

\section{Supplementary Information accompanies this paper on Cell Death and Differentiation website (http://www.nature.com/cdd)}

